Ann. Sci. forest., 1970, 27 (3), 287-301.

\title{
COMPARAISON DE DEUX GÉNÉRATIONS SUCCESSIVES DE SAPIN (ALBIES ALBA MILL.) : STRUCTURE GÉNÉTIQUE DE POPULATION, HYPOTHÈSES \\ SUR L'ACTION DE LA SÉLECTION NATURELLE
}

\author{
M. ARBEZ et C. MILLIER \\ Station d'Amélioration des Arbres forestiers, \\ Station de Biométrie, \\ Centre national de Recherches forestières, 54 - Nancy \\ Institut national de la Recherche agronomique.
}

RESUME

On a étudié en pépinière les descendances maternelles de 20 arbres d'une forêt naturelle (B) âgée de 130 à 140 ans et celles de 20 arbres de la génération suivante (R) issue de la première par semis naturel et âgée de 40 ans environ.

On a enregistré les principaux résultats suivants:

- $\mathrm{B}$ et $\mathrm{R}$ ont des poids de 1.000 graines sensiblement identiques.

- Les descendants de la population $R$ sont à la fois plus tardifs (débourrement végétatif) et plus vigoureux (dimensions, nombre de branches) que ceux de la population B.

- La variabilité inter-famille est plus forte chez B que chez R. On observe la tendance inverse pour la variabilité intra-famille. Par conséquent, les valeurs d'héritabilité de $\mathrm{B}$ sont toujours supérieures à celles de R. Dans la majorité des cas, les valeurs d'héritabilité de $\mathrm{R}$ sont d'ailleurs nulles.

- Vigueur et tardiveté du débourrement sont positivement corrélées tant au plan phénotypique (dans B et R) qu'au plan génétique (dans B seulement).

L'interprétation de ces résultats amène à faire deux types d'hypothèses, l'un concernant l'influence éventuelle de l'âge des semenciers, l'autre basé sur des modifications de structure génétique. Ce dernier type d'hypothèses explique de façon plus satisfaisante les différences enregistrées.

- Si l'âge des semenciers intervient sur la vigueur des semis, dans le cas particulier ce ne peut pas être par l'intermédiaire du poids des graines: il faut imaginer une modification de la nature biochimique des réserves de la graine ou du patrimoine eytoplasmique (héritage des chloroplastes maternels) ou encore de l'A.R.N.

- On peut interpréter l'augmentation de vigueur de $\mathrm{R}$ par rapport à $\mathrm{B}$ et la réduction correspondante de la part de variance génétique additive dans la variance totale comme un effet d'hétérosis. Cet effet d'hétérosis résulterait de la rupture des cercles de consanguinité de B provoquée par la dissémination des graines à distance. 
- On peut expliquer l'augmentation de tardiveté de $\mathrm{R}$ et la réduction correspondante de la variabilité inter-famille par le jeu d'une sélection naturelle, brutale et juvénile, en faveur des tardifs. Le site de $\mathrm{R}$ est, en effet, particulièrement propice aux gelées de printemps.

\section{I. - PRESENTATION}

Dans le but initial d'étudier l'influence de l'âge des semenciers sur les performances des semis, on a comparé en pépinière les descendances de deux générations successives d'une provenance naturelle méridionale de sapin pectiné (Abies alba Mill.).

On a récolté séparément les graines de vingt arbres de la forêt naturelle de la Betouze d'Arques (B) (France, Hautes Corbières) âgée de 130 à 140 ans et de vingt arbres appartenant à la génération suivante, issue de la première par semis naturel, et âgée de 40 ans environ. Cette dernière porte le nom de Rialsesse (R).

Ces deux populations étroitement apparentées ne sont séparées que par $100 \mathrm{~m}$ environ sur le terrain. Les arbres échantillonnés dans chacune d'elles étaient distants de 5 à 20 mètres les uns des autres.

Les premiers résultats de cette expérience ont déjà fait l'objet d'une communication à la seconde Consultation mondiale sur l'Amélioration des Arbres forestiers (Washington, 1969). Ce nouveau mémoire porte sur un nombre plus élevé de caractères et accorde une importance particulière aux problèmes de structure génétique.

\section{II. - DISPOSITIFS ET MESURES EN PEPINIERE}

\section{1. - Dispositifs}

A l'âge de 2 ans, les descendances maternelles (pollinisation libre) des 20 arbres choisis dans chacune des deux populations ont été repiquées selon des dispositifs identiques, mais séparés, en lattice rectangulaire doublé $(3 \times 2$ répétitions au total) à raison de 60 individus par descendance (10 individus par parcelle unitaire).

\section{2. - Mesures}

Cette expérience a fait l'objet de nombreuses mesures. Nous retiendrons les suivantes:

— Graines : poids de 1.000 graines estimé à partir de 4 répétitions de 25 graines non triées par descendance.

- Semis : nombre des cotylédons mesurés sur un échantillon longueur des cotylédons ( $\mathrm{ma}$, de 40 individus/descendance.

Hauteur à 2 ans $(\mathrm{cm})$ estimée au niveau individuel à partir de la différence :

hauteur totale à 3 ans - pousse terminale à 3 ans (différence des deux variables) sur les 60 individus par descendance que compte le dispositif (1).

- Plants repiqués :

Dimensions : hauteur à 3 ans $(\mathrm{cm})$,

hauteur à 4 ans $(\mathrm{cm})$,

diamètre au collet à 4 ans $(\mathrm{mm})$.

Ramification: nombre de rameaux latéraux du verticille de $3^{\mathrm{e}}$ année.

(1) Toutes les mesures suivantes portent sur le même échantillon. 
Phénologie : débourrement à 3 ans, noté sur le bourgeon terminal à l'aide d'une échelle conventionnelle à 6 classes (1).

Ces notations ont été effectuées simultanément sur les 2 disp̣ositifs, lorsque la fréquence des individus débourrés des 40 descendances avoisinait en moyenne $50 \%$. En raison de l'échelle adoptée, les notes varient dans le même sens que la précocité.

\section{III. - METHODE}

Pour chaque caractère $(2)$ et dans chaque population on a effectué :

- Une analyse de variance sur les moyennes de parcelles unitaires du dispositif en lattice rectangulaire triple doublé, correspondant aux degrés de liberté suivants (D.L.) :
Source de variation
D.L.
Répétitions

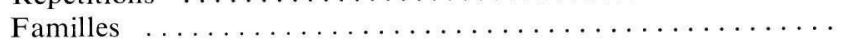

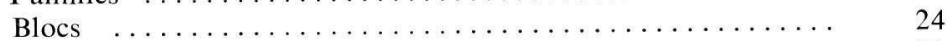

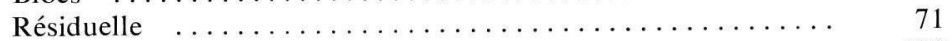

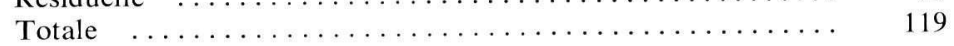

A partir de ces analyses de variance, on a procédé à une comparaison des moyennes de familles (test $F$ approché) et des moyennes générales de population (test $t$ de STUDENT).

- Une analyse de variance et de covariance à un facteur contrôlé (3) sur les données individuelles, correspondant au schéma suivant:

\begin{tabular}{ll|c|c|c|c}
\hline \hline & $\begin{array}{c}\text { Source } \\
\text { de variation }\end{array}$ & $\begin{array}{c}\text { Somme des } \\
\text { carrés }\end{array}$ & D.L. & Carré moyen \\
\hline Variance $\ldots . .$. & $\begin{array}{c}\text { Famille } \\
\text { résiduelle }\end{array}$ & $\begin{array}{c}\mathrm{SC}_{\mathrm{F}} \\
\mathrm{SC}_{\mathrm{W}}\end{array}$ & $\begin{array}{r}19 \\
1136\end{array}$ & $\begin{array}{l}\mathrm{CM}_{\mathrm{F}}=\mathrm{C}^{2} \mathrm{~W}_{\mathrm{W}}+\mathrm{kr}^{2} \sigma_{\mathrm{F}} \\
\mathrm{CM}_{\mathrm{W}}=\sigma_{\mathrm{W}}^{2}\end{array}$ \\
\hline \hline
\end{tabular}

\begin{tabular}{|c|c|c|c|c|}
\hline & $\begin{array}{l}\text { Source de } \\
\text { variation }\end{array}$ & $\begin{array}{c}\text { Somme } \\
\text { des produits }\end{array}$ & D.L. & Produit moyen \\
\hline Covariance... . & $\begin{array}{l}\text { Famille } \\
\text { résiduelle }\end{array}$ & $\begin{array}{l}\mathrm{SP}_{\mathrm{F}} \\
\mathrm{SP}_{\mathrm{W}}\end{array}$ & $\begin{array}{r}19 \\
1136\end{array}$ & $\begin{array}{l}\mathrm{CM}_{\mathrm{F}}=\mathrm{C}_{\sigma^{2}{ }_{\mathrm{w}}}+\mathrm{kr} \sigma_{\mathrm{F}}^{2} \\
\mathrm{PM}_{\mathrm{W}}=\mathrm{COV}_{\mathrm{w}}\end{array}$ \\
\hline
\end{tabular}

où $k=$ nombre d'individus par parcelle (soit en principe 10 ),

$r=$ nombre de répétitions (soit 6),

$\sigma^{2}{ }_{\mathrm{F}}$ et $\mathrm{COV}_{\mathrm{F}}=$ variance et covariance entre familles,

$\sigma^{2}{ }_{\mathrm{W}}$ et $\mathrm{COV}_{\mathrm{W}}=$ variance et covariance résiduelle (individus dans les familles).

(1) Note 1: bourgeon terminal dormant;

- 2: bourgeon terminal gonflé, vert tendre;

- 3: bourgeon terminal débourré, aiguilles apparentes serrées en pinceau;

- 4: le pinceau d'aiguilles s'allonge, les extrémités se séparent;

- 5: les premières aiguilles sont presque horizontales ;

- 6: la pousse s'allonge et la majorité des aiguilles sont horizontales.

(2) Poids de 1.000 graines excepté.

(3) Ce second schéma d'analyse de variance a été appliqué aux différents caractères sauf, le poids de 1.000 graines, le nombre et la longueur des cotylédons. 
On en déduit :

- le coefficient de corrélation intra-classe ?

$\rho=\frac{\sigma_{F}}{\sigma_{F}^{2}+\sigma^{2 x}}$ et par conséquent l'héritabilité au sens strict $h^{2}=4 \rho$

en faisant les hypothèses suivantes :

a) panmixie,

b) aucun effet d'environnement commun pour les individus de la même descendance,

c) descendances maternelles issues de pollinisation libre, constituées de demi-frères $\sigma^{2}{ }_{\mathrm{F}}$ estime $1 / 4$ de la variance génétique additive).

Ces hypothèses ne sont sans doute vérifiées qu'incomplètement.

même

- la corrélation moyenne entre deux caractères $\mathrm{X}$ et $\mathrm{Y}$ sur les individus d'une ille :

$$
r_{\mathrm{W}}=\frac{\operatorname{COV}_{\mathrm{W}}(\mathrm{X}, \mathrm{Y})}{\sqrt{\sigma^{2}{ }_{\mathrm{W}}\left({ }_{\mathrm{X}}\right) \times \sigma_{\mathrm{W}}(\mathrm{Y})}}
$$

- la corrélation génétique :

$$
r_{\mathrm{A}}=\frac{\operatorname{COV}_{\mathrm{F}}(\mathrm{X}, \mathrm{Y})}{\sqrt{\sigma^{2}{ }_{\mathrm{F}}\left({ }_{\mathrm{X}}\right) \times \sigma^{2}{ }_{\mathrm{F}}\left({ }_{\mathrm{Y}}\right)}}
$$

La variabilité inter-famille a été estimée par la variance $\sigma^{2}{ }_{\mathrm{F}}$ de la distribution des moyennes de familles autour de la moyenne générale de chaque population.

La variabilité intra-famille, c'est-à-dire la variance moyenne de la distribution des valeurs individuelles autour de la moyenne de chaque famille, a été estimée par $\sigma^{2}{ }_{W}$ tiré de l'analyse de variance à un facteur contrôlé.

\section{IV. - RESULTATS}

Les résultats concernant moyennes, variances et héritabilités figurent au tableau 1.

\section{1. - Moyennes générales de population}

$\mathrm{B}$ et $\mathrm{R}$ ont des poids de 1000 graines sensiblement identiques.

Les nombres de cotylédons sont très voisins, par contre les longueurs sont plus élevées chez $\mathbf{R}$ que chez $\mathrm{B}$.

Les dimensions des semis et des plants de $\mathrm{R}$ sont toujours supérieures à celles de $\mathrm{B}$. Il en va de même pour le nombre des rameaux latéraux.

Le débourrement de $\mathrm{R}$ est plus tardif que celui de $\mathrm{B}$.

\section{2. - Variabilité inter-famille dans chaque population}

Dans l'ensemble, la variabilité inter-famille est plus accusée chez B que chez R. La comparaison des coefficients de variation correspondants est particulièrement nette. Les analyses de variance effectuées sur les données des dispositifs en pépinière montrent que les familles de $\mathrm{R}$ sont remarquablement homogènes, il n'existe entre elles aucune différence significative sur l'ensemble des caractères étudiés. 


\section{3. - Variabilité intra-famille (individuelle) dans chaque population}

En moyenne, la variabilité intra-famille de $\mathrm{R}$ est très légèrement supérieure à celle de $\mathbf{B}$ pour la plupart des caractères étudiés.

\section{4. - Héritabilité}

Excepté pour le nombre des cotylédons, les valeurs d'héritabilité de $\mathbf{R}$ sont pratiquement nulles. Celles de B sont toujours nettement supérieures, mais excepté pour le nombre des rameaux $(0,60)$ et le débourrement $(0,41)$, ces valeurs demeurent faibles en valeur absolue. Par ailleurs, ces valeurs supposent que les descendances maternelles sont essentiellement constituées de demi-frères. En fait, il s'agit plus probablement d'un mélange de demi-frères et de pleins-frères (1) et les valeurs réelles d'héritabilité doivent être plus faibles.

\section{5. - Corrélations entre caractères}

Corrélations au niveau des moyennes de familles.

L'étude des corrélations entre caractères mesurés avant et après repiquage ne peut être faite qu'à ce niveau. En se limitant à cet aspect: le poids de 1000 graines n'est corrélé qu'avec le nombre $(0,603 * *)$ et la longueur $(0,543 *)$ des cotylédons, mais uniquement dans la population $\mathrm{B}$, par contre dans les deux populations le nombre et la longueur des cotylédons sont positivement corrélés $(\mathbf{B}=0,517 *, \mathbf{R}=0,538 *)$.

Dans cette expérience, les caractères des graines et des semis (hauteur à 2 ans exceptée) ne sont donc pas corrélés avec les caractères mesurés à 3 et 4 ans.

Corrélations au niveau des individus dans les familles.

Il s'agit des coefficients de corrélation $r_{w}$ définis au chapitre 3 . Les résultats font l'objet du tableau 2. Le schéma des liaisons est pratiquement le même dans les deux populations :

- Entre caractères dimensionnels on observe un fléchissement des liaisons entre 3 et 4 ans. Tout se passe comme si les effets de la crise d'installation ne s'étaient pas manifestés immédiatement après le repiquage mais seulement un an plus tard. En fait, la pousse terminale de l'année est surtout influencée par les conditions de nutrition de l'année $n-1$ (préformation de la pousse dans le bourgeon terminal); il est donc normal de constater que les perturbations succédant normalement au repiquage en $3^{*}$ année de végétation n'affectent que l'année suivante les mesures de pousse et de hauteur totale.

- Le nombre des rameaux du verticille de $3^{\circ}$ année est plus fortement lié au diamètre au collet qu'aux diverses hauteurs. Ce caractère de ramification semble conditionné de toute façon par le niveau de vigueur général des individus.

- La précocité du débourrement est corrélée négativement avec le nombre des rameaux latéraux et les dimensions des individus. En ce qui concerne les hauteurs,

(1) C'est ce gui ressort de l'analyse de nombreux tests de descendances maternelles réalisés sur diverses espèces d'arbres forestiers. 


\section{TABLEAU \\ Moyennes, variances, héritabil}

\begin{tabular}{|c|c|c|c|}
\hline $\begin{array}{l}\text { Caractère } n^{\circ}: \\
\left(\text { Character } n^{0}:\right)\end{array}$ & $\begin{array}{l}\text { Population } \\
\text { (population) }\end{array}$ & $\begin{array}{l}\text { Moyenne } \\
\text { générale de } \\
\text { population } \\
\text { (General mean } \\
\text { of population) }\end{array}$ & $\begin{array}{l}\text { Comparaison des } \\
\text { familles dans chaque } \\
\text { population (1) } \\
\text { F (19,71) comparison } \\
\text { of progenies within } \\
\text { each population (1) }\end{array}$ \\
\hline $\begin{array}{l}1 \text { Poids de } 1.000 \text { graines }(\mathrm{g}) \\
\text { (Weight of } 1000 \text { seeds) }\end{array}$ & $\begin{array}{cl} & \mathrm{B} \\
& \mathrm{R} \\
\text { test de } & \text { comparaison }\end{array}$ & $\begin{aligned} & 69,10 \\
& 69,65 \\
t_{3 \mathrm{~S}}= & 0,08 \mathrm{NS}(4)\end{aligned}$ & \\
\hline $\begin{array}{l}2 \text { Nombre des cotylédons (Num- } \\
\text { ber of cotylédons) } \ldots \ldots \ldots \ldots\end{array}$ & $\begin{array}{lll} & & \mathrm{B} \\
& \mathrm{R} \\
\text { test de } & \text { comparaison } \\
\end{array}$ & $\begin{array}{c}5,09 \\
5,03 \\
t_{8 .}=2,21 *\end{array}$ & $\begin{array}{c}2,36 * * \\
1,52 \mathrm{NS} \quad(5)\end{array}$ \\
\hline $\begin{array}{l}3 \text { Longueur des cotylédons }(\mathrm{mm}) \\
\text { (Length of cotylédons) } \ldots \ldots\end{array}$ & 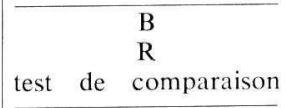 & $\begin{aligned} & 25,25 \\
& 25,91 \\
t_{82}= & 4,54 * *\end{aligned}$ & $\begin{array}{c}3,04 * * \\
0,96 \mathrm{NS}(5)\end{array}$ \\
\hline $\begin{array}{l}4 \text { Hauteur à } 2 \text { ans }(\mathrm{cm}) \text { (Height } \\
\text { at } 2 \text { years) } \ldots \ldots \ldots \ldots \ldots \ldots\end{array}$ & $\begin{array}{lll} & & \mathrm{B} \\
& \mathrm{R} \\
\text { test } & \text { de } & \text { comparaison } \\
\end{array}$ & $\begin{array}{c}7,18 \\
7,60 \\
t_{142}=4,95 * *\end{array}$ & $\begin{array}{l}2,14 * * \\
0,67 \mathrm{NS}\end{array}$ \\
\hline $\begin{array}{l}5 \text { Hauteur à } 3 \text { ans }(\mathrm{cm})(\text { Height } \\
\text { at } 3 \text { years }) \ldots \ldots \ldots \ldots \ldots\end{array}$ & $\begin{array}{ll} & \mathrm{B} \\
& \mathrm{R} \\
\text { test de } & \text { comparaison } \\
\end{array}$ & $\begin{array}{c}11,54 \\
12,60 \\
t_{142}=10,7 * *\end{array}$ & $\begin{array}{l}1,59 \mathrm{NS} \\
0,74 \mathrm{NS}\end{array}$ \\
\hline $\begin{array}{l}6 \text { Hauteur à } 4 \text { ans }(\mathrm{cm})(\text { Height } \\
\text { at } 4 \text { years }) \ldots \ldots \ldots \ldots \ldots\end{array}$ & $\begin{array}{lll} & \mathrm{B} \\
& \mathrm{R} \\
\text { test de } & \text { comparaison }\end{array}$ & $\begin{array}{c}21,02 \\
22,56 \\
t_{142}=6,66 * *\end{array}$ & $\begin{array}{l}2,20 * * \\
1,24 \mathrm{NS}\end{array}$ \\
\hline 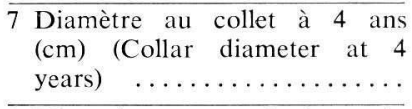 & $\begin{array}{lll} & & \mathrm{B} \\
& \mathrm{R} \\
\text { test de } & \text { comparaison }\end{array}$ & $\begin{array}{c}9,48 \\
10,29 \\
t_{142}=11,6 * *\end{array}$ & $\begin{array}{l}3,68 * * \\
0,85 \mathrm{NS}\end{array}$ \\
\hline 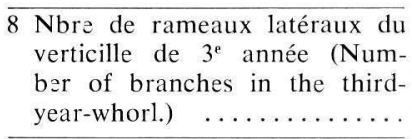 & \begin{tabular}{|cl} 
& $\mathrm{B}$ \\
& $\mathrm{R}$ \\
test de & comparaison
\end{tabular} & $\begin{array}{c}1,62 \\
2,06 \\
t_{142}=16,8 * *\end{array}$ & $\begin{array}{l}10,07 * * \\
1,20 \mathrm{NS}\end{array}$ \\
\hline $\begin{array}{l}9 \text { Débourrement à } 3 \text { ans (nota- } \\
\text { tion) (Note of flushing at } 3 \\
\text { years) } \quad \ldots \ldots \ldots \ldots \ldots \ldots \ldots \ldots \ldots\end{array}$ & $\begin{array}{ll} & \mathrm{B} \\
& \mathrm{R} \\
\text { test de comparaison }\end{array}$ & $\begin{array}{c}2,46 \\
2,21 \\
\iota_{142}=9,73 * *\end{array}$ & $\begin{array}{l}6,27 * * \\
0,63 \mathrm{NS}\end{array}$ \\
\hline
\end{tabular}

(1) Résultats des analyses de variance en lattice rectangulaire doublé [Results from analys

(2) Distribution des moyennes de familles autour de la moyenne générale de chaque populatio

(3) Tirée de l'analyse de variance à un facteur contrôlé (from results of one way analysis

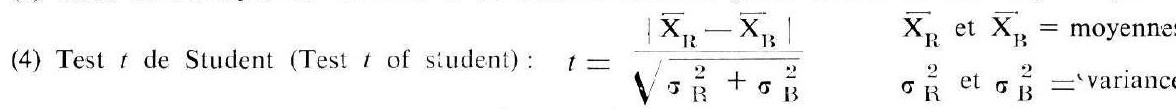

- Pour le poids de 1.000 graines $\sigma \frac{2}{\mathrm{R}}$ et $\sigma \frac{2}{3}$ ont été estimés directement à partir de de degrés de liberté de $t$ égal à $\left(20^{\circ}-1\right)+(20-1)=38$.

- Pour les autres caractères, $\sigma \frac{2}{3}$ et $\sigma \frac{2}{3}$ ont été estimés à partir des variances résiduell par le nombre total de parcelles unitaires de chaçue dispositif (nombre des moyennes élémentair de $t$ égal à la somme des degrés de liberté des variances résiduelles des deux dispositifs so autres caractères.

(5) 19 et 41 degrés de liberté. En effet, ces deux caractères n'ont été mesurés que sur 2 rép 41 degrees of freedom: these two characters have been measured on only two replications of eac 
Table 1

ns, variances, heritabilities

\begin{tabular}{|c|c|c|c|c|}
\hline $\begin{array}{l}\text { Variance bru } \\
\text { inter-famille } \\
\text { (between-prog } \\
\text { variance) }(2\end{array}$ & & \multicolumn{2}{|c|}{$\begin{array}{l}\text { Variance résiduelle } \\
\text { (intra-famille } \\
\text { ou individuelle) (3) } \\
\text { (within-progeny } \\
\text { variance) (3) }\end{array}$} & $\begin{array}{l}\text { Héritabilité } \\
\text { (Heritability) } \\
\mathrm{h} 2=\frac{4 \sigma^{2}{ }_{\mathrm{F}}}{\sigma^{2}{ }_{\mathrm{F}}+\sigma^{2} \mathrm{~W}}\end{array}$ \\
\hline$\sigma^{2}$ & $\mathrm{CV}_{13} \%$ & $\sigma^{2} w$ & $\mathrm{CV}_{\mathrm{W}} \%$ & \\
\hline $\begin{array}{c}44,43 \\
5,65 \\
F(19,19)=7,86 * *\end{array}$ & $\begin{array}{l}9,7 \\
3,4\end{array}$ & & & \\
\hline $\begin{array}{c}0,01 \rightarrow 5 \\
0,0098 \\
\mathrm{~F}(19,19)=1,99 \mathrm{NS}\end{array}$ & $\begin{array}{l}2,7 \\
2,0\end{array}$ & $\begin{array}{c}0,3290 \\
0,2610 \\
F(761,761)=1,26 * *\end{array}$ & $\begin{array}{l}11,3 \\
10,2\end{array}$ & $\begin{array}{l}0,31 \\
0,21\end{array}$ \\
\hline $\begin{array}{c}0,7967 \\
0,2198 \\
F(19,19)=3,62 * *\end{array}$ & $\begin{array}{l}3,5 \\
1,8\end{array}$ & $\begin{array}{c}6,9910 \\
8,8211 \\
F(720,720)=1,26 * *\end{array}$ & $\begin{array}{l}10,5 \\
11,5\end{array}$ & $\begin{array}{l}0,33 \\
0,00\end{array}$ \\
\hline $\begin{array}{l}0,1894 \\
0,1460 \\
F(19,19)=1,30 \mathrm{NS}\end{array}$ & $\begin{array}{l}6,1 \\
5,0\end{array}$ & $\begin{array}{c}3,8011 \\
3,7100 \\
\mathrm{~F}(1146,1136)=1,02 \mathrm{NS}\end{array}$ & $\begin{array}{l}27,2 \\
25,4\end{array}$ & $\begin{array}{l}0,10 \\
0,00\end{array}$ \\
\hline $\begin{array}{c}0,3262 \\
0,1546 \\
\mathrm{~F}(19,19)=2,11 \mathrm{NS}\end{array}$ & $\begin{array}{l}4,9 \\
3,1\end{array}$ & $\begin{array}{c}4,9693 \\
6,4394 \\
\mathrm{~F}(1146,1136)=1,30 \mathrm{NS}\end{array}$ & $\begin{array}{l}19,3 \\
20,2\end{array}$ & $\begin{array}{l}0,12 \\
0,01\end{array}$ \\
\hline $\begin{array}{c}1,0872 \\
0,7343 \\
\mathrm{~F}(19,19)=1,48 \mathrm{NS}\end{array}$ & $\begin{array}{l}4,9 \\
3,8\end{array}$ & $\begin{array}{r}14,5371 \\
26,4918 \\
F(1146,1136)=1,82 \cdots\end{array}$ & $\begin{array}{l}18,1 \\
22,8\end{array}$ & $\begin{array}{l}0,18 \\
0,04\end{array}$ \\
\hline $\begin{array}{c}0,1534 \\
0,0504 \\
\mathrm{~F}(19,19)=3,04 * *\end{array}$ & $\begin{array}{l}4,1 \\
2,2\end{array}$ & $\begin{array}{r}2,1025 \\
3,2101 \\
F(1146,1135)=1,53 * *\end{array}$ & $\begin{array}{l}15,3 \\
17,4\end{array}$ & $\begin{array}{l}0,22 \\
0,00\end{array}$ \\
\hline $\begin{array}{c}0,0596 \\
0,0111 \\
\mathrm{~F}(19.19)=6.27 * *\end{array}$ & $\begin{array}{r}16,3 \\
5,1\end{array}$ & $\begin{array}{r}0,3772 \\
0,4954 \\
F(1146,1136)=1,32 * *\end{array}$ & $\begin{array}{l}37,9 \\
34,2\end{array}$ & $\begin{array}{l}0,60 \\
0,03\end{array}$ \\
\hline $\begin{array}{c}0,0438 \\
0,0041 \\
F(19,19)=10,68 *\end{array}$ & $\begin{array}{l}8,5 \\
2,7\end{array}$ & $\begin{array}{c}0,3157 \\
0,2933 \\
F(1146,1136)=1,08\end{array}$ & $\begin{array}{l}22,8 \\
24,5\end{array}$ & $\begin{array}{l}0,41 \\
0,00\end{array}$ \\
\hline
\end{tabular}

of variance (rectangular lattice $\times 2$ )].

(Distribution of progenies means around the general mean of each population).

variance).

générales de population (general means of population).

de $\bar{X}_{\mathrm{R}}$ et $\bar{X}_{\mathrm{B}}$ (variances of $\mathrm{X}_{\mathrm{R}}$ and $\mathrm{X}_{\mathrm{B}}$ ).

variance de la distribution des moyennes de descendances de chaque population, d'où un nombre

des deux dispositifs en lattice rectangulaire doublé, en divisant simplement ces variances résiduelles ayant effectivement servi au calcul de la moyenne générale). D'où un nombre de degrés de liberté $41+41=82$ pour le nombre et la longueur des cotylédons (voir 5) et $71+71=142$ pour les titions de chaque lattice rectangulaire, soit au total 4 répétitions pour le dispositif doublé (19 and rectangular lattice, then on the total four replications for the double rectangular lattice). 
Limites de signification pour 1.000 degrés de liberté (1) au seuil de probabilité $5 \%: 0,062 *$ : au seuil de probabilité $1 \%: 0,081 \% *$, (Limit of significance for 1.000 degrees of freedom (1) at the $5 \%$ seuil de probabilité $1 \%: 0,081 *$, (Limit of significance for 1.000 degrees of

[Correlation coefficients between characters at the individual level within progenies $\left.\left(r_{\mathrm{w}}\right)\right]$.

Les valeurs non significatives sont remplacées par des tirets (Non significant values are replaced by (iashes).

\begin{tabular}{|c|c|c|c|c|c|c|c|}
\hline & & 4 & 5 & 6 & 7 & 8 & 9 \\
\hline $\begin{array}{l}4 \text { Hauteur à } 2 \text { ans } \ldots \ldots \ldots \ldots \ldots \ldots \ldots \ldots \ldots \ldots \ldots \ldots \ldots \ldots \\
\text { (Height at } 2 \text { years) } \ldots \ldots \ldots \ldots \ldots \ldots \ldots \ldots\end{array}$ & $\begin{array}{l}\mathrm{B} \\
\mathrm{R}\end{array}$ & $\begin{array}{l}1,000 \\
1,000\end{array}$ & $\begin{array}{l}0,819 * * \\
0,778 * *\end{array}$ & $\begin{array}{l}0,352 * * \\
0,424 * *\end{array}$ & $\begin{array}{l}0,334 * * \\
0,456 * *\end{array}$ & $\begin{array}{l}0,181 * \\
0,191 * *\end{array}$ & $\begin{array}{l}-0,202 * * \\
-0,215 * *\end{array}$ \\
\hline $\begin{array}{l}5 \text { Hauteur à } 3 \text { ans } \ldots \ldots \ldots \ldots \ldots \ldots \ldots \ldots \ldots \ldots \ldots \ldots \ldots \ldots \ldots \ldots \ldots \ldots \ldots \ldots \ldots \ldots \\
\text { (Height at } 3 \text { years }) \ldots \ldots \ldots \ldots \ldots \ldots\end{array}$ & $\begin{array}{l}\mathrm{B} \\
\mathrm{R}\end{array}$ & $\begin{array}{l}0,819 * * \\
0,778 * *\end{array}$ & $\begin{array}{l}1,000 \\
1,000\end{array}$ & $0,572 * *$ & $\begin{array}{l}0,525 * * \\
0,605 * *\end{array}$ & $0,146^{* *}$ & $\begin{array}{l}-0,072 \% \\
-0,087 \%\end{array}$ \\
\hline $\begin{array}{l}6 \text { Hauteur à } 4 \text { ans } \ldots \ldots \ldots \ldots \ldots \ldots \ldots \ldots \ldots \ldots \ldots \ldots \ldots \ldots \ldots \ldots \ldots \\
\text { (Height at } 4 \text { years) } \ldots \ldots \ldots \ldots \ldots \ldots \ldots \ldots\end{array}$ & $\begin{array}{l}\mathrm{B} \\
\mathrm{R}\end{array}$ & $\begin{array}{l}0,352 * * \\
0,424 * *\end{array}$ & $\begin{array}{l}0,572 * * \\
0,653 * *\end{array}$ & $\begin{array}{l}1,000 \\
1,000\end{array}$ & $\begin{array}{l}0,490 * * \\
0,579 * *\end{array}$ & $0,142 *$ & - \\
\hline $\begin{array}{l}7 \text { Diamètre au collet à } 4 \text { ans } \ldots \ldots \ldots \ldots \ldots \ldots \\
\text { (Co'ar diameter at } 4 \text { years }) \ldots \ldots \ldots \ldots \ldots\end{array}$ & $\begin{array}{l}\mathrm{B} \\
\mathrm{R}\end{array}$ & $\begin{array}{l}0,334 * * \\
0,456 * *\end{array}$ & $\begin{array}{l}0,525 * * \\
0,605 * *\end{array}$ & $\begin{array}{l}0,490 * * \\
0,579 * *\end{array}$ & $\begin{array}{l}1,000 \\
1,000\end{array}$ & $\begin{array}{l}0,366 * * \\
0,266 * *\end{array}$ & $\begin{array}{l}-0,100 * * \\
-0,102 * *\end{array}$ \\
\hline $\begin{array}{l}8 \mathrm{Nb} \text {. rameaux lat. } 3^{e} \text { année } \ldots \ldots \ldots \ldots \ldots \ldots \ldots \\
\text { (Number of branches in the third-year-whorl) } \ldots\end{array}$ & $\begin{array}{l}\mathrm{B} \\
\mathrm{R}\end{array}$ & $\begin{array}{l}0,181^{\mathrm{e} * *} \\
0,191^{4 * *}\end{array}$ & $0.146^{* * *}$ & $\begin{array}{c}0,142^{\mathrm{e} * * *} \\
-\end{array}$ & $0,366 * *$ & $\begin{array}{l}1,000 \\
1,000\end{array}$ & $\begin{array}{l}-0,550 * * \\
-0,483 * *\end{array}$ \\
\hline $\begin{array}{l}9 \text { Débourrement à } 3 \text { ans } \ldots \ldots \ldots \ldots \ldots \ldots \ldots \\
\text { (Note of flushing at } 3 \text { years) } \ldots \ldots \ldots \ldots \ldots \ldots\end{array}$ & $\begin{array}{l}\mathrm{B} \\
\mathrm{R}\end{array}$ & $\begin{array}{l}-0,202 * * \\
-0,215 * *\end{array}$ & $\begin{array}{l}-0,072 * \\
-0,087 * *\end{array}$ & - & $\begin{array}{l}-0,100 * * \\
-0.102 * *\end{array}$ & $\begin{array}{l}-0.550 * * \\
-0,483 * *\end{array}$ & $\begin{array}{l}1,000 \\
1,000\end{array}$ \\
\hline
\end{tabular}


la liaison est maximale pour la hauteur à 2 ans, elle diminue pour la hauteur à 3 ans et s'annule pour la hauteur à 4 ans. La liaison est donc maximale avec la hauteur des individus au moment des observations de débourrement, au printemps de la $3^{\text {e }}$ année. Ce qui revient à dire qu'à cet âge les individus les plus grands étaient aussi les plus tardifs.

\section{Corrélations génétiques.}

Le coefficient $r_{\mathrm{A}}$ défini au chapitre 3 exprime la corrélation entre valeurs reproductrices ( breeding values»). C'est donc la notion la plus utile lorsqu'on recherche une amélioration génétique portant sur plusieurs caractères simultanément en utilisant la sélection individuelle et les mécanismes de reproduction sexuée.

L'intervalle de confiance correspondant à l'estimation de $r_{\mathrm{A}}$ augmente lorsque l'héritabilité des deux caractères en cause diminue (REEve, 1955). De fait, lorsque l'héritabilité des deux caractères est faible ou nulle, la précision de l'estimation de $r_{\mathrm{A}}$ devient dérisoire. C'est le cas de la population $\mathbf{R}$ où les valeurs trouvées s'échelonnent entre zéro (corrélations faisant intervenir le diamètre au collet et le débourrement) et des valeurs supérieures à 1 (corrélations entre le nombre de rameaux latéraux et les hauteurs). Dans ces conditions, nous nous sommes bornés à l'étude de la matrice des corrélations génétiques pour la seule population $\mathbf{B}$, reproduite au tableau 3 .

Les diverses caractéristiques dimensionnelles sont assez fortement liées entre elles et avec le nombre des rameaux latéraux. Nous avons par ailleurs la preuve que, dans la population $\mathrm{B}$, la précocité du débourrement et la vigueur sont négativement corrélées au plan génétique. Dans ce cas, il est parfaitement possible d'entreprendre une sélection combinée pour la tardiveté du débourrement et la vigueur, deux critères particulièrement intéressants pour l'amélioration génétique du sapin.

\section{V. - INTERPRETATION}

A quoi peut-on attribuer les différences enregistrées entre les descendances des populations $\mathrm{B}$ et $\mathrm{R}$ ? A priori, deux catégories d'explications peuvent être avancées, la première fondée sur la différence d'âge entre les semenciers, la seconde faisant intervenir une modification de structure génétique. Ces deux types d'explication seront successivement examinés.

\section{1. - Influence de l'âge des semenciers}

On ne décèle aucune liaison entre le poids des graines et la vigueur des semis ( $\$ 4.5$ : corrélation au niveau des moyennes de familles). Comme par ailleurs le poids des graines est pratiquement identique dans les deux populations, on en conclut qu'il est incapable d'expliquer leur différence de hauteur (1).

(1) On pourrait objecter qu'il s'agit du poids des graines non triées. En fait chez le sapin, les graines pleines et les graines vides ont des poids très voisins (le tri densimétrique est très peu précis). Dans chaque population, il n'y a d'ailleurs aucune corrélation entre le poids des graines non triées des descendances et le pourcentage de graines pleines correspondant. 
TABleaU 3. TABle 3

Coefficients de corrélation génétique $\left(r_{\mathrm{A}}\right)$ pour la population $B$

Genetic correlation coefficients $\left(r_{1}\right)$ in the population $B$

\begin{tabular}{|c|c|c|c|c|c|c|}
\hline $\begin{array}{c}\text { Caractère } n^{\circ} \\
\left(\text { Character } n^{\circ}\right)\end{array}$ & 4 & 5 & 6 & 7 & 8 & 9 \\
\hline $\begin{array}{l}4 \text { Hauteur à } 2 \text { ans } \ldots \ldots \ldots \ldots \\
\text { (Height at } 2 \text { years) } \ldots \ldots \ldots \ldots\end{array}$ & 1,000 & 0,912 & 0,001 & 0,819 & 0865 & -0.709 \\
\hline $\begin{array}{l}5 \text { Hauteur à } 3 \text { ans } \ldots \ldots \ldots \\
\text { (Height at } 3 \text { years) } \ldots \ldots \ldots \ldots\end{array}$ & 0,912 & 1,000 & 0,783 & 0,885 & 6.854 & $-0.57 \pi$ \\
\hline $\begin{array}{l}6 \text { Hauteur à } 4 \text { ans } \ldots \ldots \ldots \ldots \\
\text { (Height at } 4 \text { years) } \ldots \ldots \ldots \ldots\end{array}$ & 0,601 & 9,783 & 1,090 & 0,654 & $1,7+7$ & $\ldots .176$ \\
\hline $\begin{array}{l}7 \text { Diamètre au collet à } 4 \text { ans } \ldots \\
\text { (Collar diameter at } 4 \text { years) } \ldots\end{array}$ & $0,31 \%$ & 0,885 & 0,654 & 1,000 & 1) 673 & $-0,231$ \\
\hline $\begin{array}{l}8 \mathrm{Nb} \text {. rameaux lat. du verticille } \\
\text { de } 3^{\mathrm{e}} \text { année } \ldots \ldots \ldots \ldots \ldots \ldots \\
\text { (Number of branches in the } \\
\text { third-year-whorl.) } \ldots \ldots \ldots \ldots \ldots\end{array}$ & 0,865 & 0,854 & 0,747 & 6,678 & 1.000 & $-0,33 ;$ \\
\hline $\begin{array}{l}9 \text { Débourrement à } 3 \text { ans } \ldots \ldots \\
\text { (Note of flushing at } 3 \text { years) } \ldots\end{array}$ & $-0,799$ & $-0,577$ & $-0,476$ & $-0,231$ & $-0,838$ & 1,000 \\
\hline
\end{tabular}


En toute rigueur, rien ne s'oppose cependant à ce que l'âge des semenciers soit malgré tout intervenu sur la vigueur des semis mais par une voie différente. En particulier on pourrait imaginer des modifications dans la nature biochimique des réserves de la graine ou même dans le patrimoine cytoplasmique (héritage des chloroplastes maternels) ou l'A.R.N.

\section{2. - Modifications de structure génétique}

Deux hypothèses peuvent être formulées pour expliquer les modifications de la structure génétique de la jeune population $(\mathrm{R})$ par rapport à la population parentale $(\mathrm{B})$ :

a) En forêt naturelle, on admet généralement que l'existence de pollinisateurs préférentiels et la dissémination des graines à faible distance entraînent une certaine consanguinité de position («cercles de voisinage »). C'est vraisemblablement ce qui se passe dans la population B : petite population naturelle relique subsistant depuis très longtemps sur une surface limitée (10 ha environ). Par contre, en ce qui concerne R, la colonisation naturelle massive des friches situées en lisière de forêt (à la faveur d'une année de fructification abondante) se traduit par la rupture des cercles de voisinage: la dissémination des graines à distance fait que deux individus voisins, issus de graines ayant germé côte à côte, n'ont plus qu'une probabilité insignifiante d'être apparentés. Un effet de vigueur hybride (hétérosis) peut alors se manifester en $\mathrm{F}_{1}{ }^{\text {(1) }}$. La configuration des peuplements et les renseignements communiqués par les forestiers locaux permettent d'admettre que $\mathrm{R}$ est bien la régénération naturelle de $\mathrm{B}$. Dans ces conditions, l'hypothèse précédente expliquerait que les descendances de $\mathbf{R}$ aient une vigueur moyenne supérieure à celle de $\mathrm{B}$, en particulier en ce qui concerne le diamètre et la hauteur.

La diminution spectaculaire et systématique des valeurs d'héritabilité de $\mathbf{B}$ à $\mathbf{R}$ renforce la thèse de l'hétérosis (2). Elle exprime en effet la réduction de la part de variance génétique additive dans la variance totale. Effectivement, $\sigma^{2}{ }_{F}$ diminue bien de $\mathrm{B}$ à $\mathrm{R}$ tandis que la variance intra-famille $\sigma^{2}{ }_{w}$ marque une légère tendance à l'augmentation.

La part des variations non génétiques restant a priori identique dans les 2 populations ${ }^{(3)}$, l'augmentation de $\sigma^{2}{ }_{W}$ traduirait essentiellement celle des effets génétiques non additifs (dominance, épistasie) (4).

Dans cette hypothèse, la différence de structure génétique entre $B$ et $R$ proviendrait donc d'une modification de l'un des éléments constituant le système de reproduction de la population (reproduction sur place en phase climax avec cercles de consanguinité et reproduction en phase pionnière avec dissémination des grains à distance et implantation aléatoire).

(1) On en connaît déjà quelques exemples chez le sapin (Arbez, 1969) et l'épicéa (LaCAZE, comrnunication personnelle) au stade juvénile.

(2) Si la diminution des valeurs d'héritabilité n'affectait que quelques caractères génétiquement correlés, elle s'expliquerait plus logiquement en supposant qu'il y a eu sélection entre B et $R$.

(3) Les dispositifs, le milieu et le mode d'élevage sont identioues dans les deux expériences; les mesures sont effectuées simultanément et de la mềme façon par les mêmes observateurs.

(4) En rapport avec l'augmentation du nombre des pères non apparentés (rupture des cercles de consanguinité, et apport supplémentaire du pollen de B sur R situé immédiatement en contre-bas). 
b) Les différences de comportement au débourrement enregistrées entre les descendances de $\mathbf{R}$ et celles de B peuvent s'expliquer par le jeu d'une sélection naturelle juvénile en faveur des tardifs. En effet, les arbres de R, dont provenaient les graines utilisées dans cette expérience, sont tous situés au creux d'un vallon étroit ouvert à l'est. Un tel site favorise les coulées d'air froid et augmente les risques de gelées tardives («trou à gelée »). Au contraire, les arbres échantillonnés dans B sont situés sur un versant nord uniforme, à l'abri de tels risques. On peut donc imaginer que lors de son installation, la population $\mathrm{R}$ a subi une sélection sévère et précoce (au stade jeune semis) en faveur de la tardiveté du débourrement. De te's phénomènes de sélection naturelle rapide en forêt ont déjà été démontrés, en particulier par BARBEr et JACKSON (1957), pour des eucalyptus de Tasmanie.

Cette hypothèse permet d'expliquer à la fois le gain de tardiveté obtenu sur la moyenne des descendances de $\mathrm{R}$ et la réduction significative de la variabilité interdescendances.

De plus, la corrélation génétique significative qui existe entre la tardiveté et la vigueur des individus de $\mathrm{B}$ peut être à l'origine d'une sélection indirecte pour la vigueur (1).

Les deux séries d'hypothèses évoquées dans le cadre d'une modification de la structure génétique : rupture des cercles de consanguinité et sélection juvénile en faveur des tardifs ne sont pas exclusives mais complémentaires. Ces hypothèses expliquent mieux les différences enregistrées entre $\mathrm{B}$ et $\mathrm{R}$ que celle faisant intervenir l'influence éventuelle de l'âge des semenciers.

\section{CONCLUSION}

Cette brève étude attire l'attention sur l'existence possible d'une évolution naturelle brutale mais localisée de certaines populations forestières sous l'action d'une pression de sélection intense exercée lors de la colonisation de zones micro-écologiques particulières.

En matière d'étude de provenances, cette conclusion amène à prendre plus de précautions dans la représentation de chaque population, en récoltant notamment les graines sur des arbres, en nombre suffisant, éloignés les uns des autres, mais répartis de façon à tenir compte des variations écologiques existantes.

L'héritabilité du débourrement jugée sur les descendances de B permet de penser qu'une sélection d'arbres tardifs en forêt naturelle peut conduire à un gain appréciable de tardiveté ${ }^{(2)}$, qu'on se contente de récolter les graines sur les arbres sélectionnés ou qu'on les utilise pour constituer un verger à graines. Par ailleurs, la sélection pour la tardiveté se traduit normalement par une sélection indirecte pour la vigueur (corrélation génétique positive tardiveté-croissance).

(1) Au moins phénotypiquement, il semblerait que la corrélation individuelle tardiveté-vigueur soit positive ou nulle dans la plupart des populations de sapin (Arbez, 1969) et d'épicéa (LACAZE, 1969) étudiées jusqu'ici.

(2) On doit néanmoins se souvenir au'en raison de l'accentuation des biais dus à des effets de milieu, l'héritabilité en forêt est probablement plus faible que celle enregistrée en milieu semicontrôlé. 
En admettant l'hypothèse d'une rupture des cercles de consanguinité dans les peuplements artificiels de $1^{\text {re }}$ génération et l'effet d'hétérosis qui peut en résulter, à la $2^{\mathrm{e}}$ génération, à qualité phénotypique égale on peut conseiller d'utiliser ces peuplements comme source de graines de préférence aux peuplements naturels. A l'inverse, il pẹt sembler dangereux de sélectionner des arbres «plus » pour la croissance dans les peuplements artificiels de $2^{e}$ génération, la supériorité de ces derniers pouvant résulter surtout d'effets d'hérédité non additive (superdominance). Ces remarques s'appliquent principalement aux espèces forestières climatiques (exigences écologiques strictes, régénération sur place). Le problème est vraisemblablement plus complexe chez les espèces pionnières (dissémination des graines à distance et colonisation des nouveaux territoires).

Reçu pour publication en juillet 1970.

\section{REMERCIEMENTS}

Que tous ceux qui ont contribué à la réalisation de ce travail trouvent ici l'expression de nos sincères remerciements :

- M. Champagnat, Professeur de Physiologie végétale à la Faculté des Sciences de Clermont-Ferrand, MM. Galoux et Nanson de la Station de Recherches des Eaux et Forêts de Belgique, pour leur étude critique du manuscrit.

- Le personnel de la Station de Biométrie du C.N.R.F. pour la réalisation des calculs.

- Le Service forestier local pour la récolte des graines.

\section{SUMMARY}

COMPARISON BETWEEN TWO SUCCESSIVE GENERATIONS OF EUROPEAN SILVER FIR

(« ABIES ALBA» MILL.) : GENETICAL STRUCTURE OF POPULATIONS AND HYPOTHESIS ABOUT THE ACTION OF NATURAL SELECTION

The maternal progenies of 20 trees from a natural forest (B) (130-140 years old) and of 20 trees from the next generation (R) by natural sowing (40 years old) were studied at nursery stage.

Results about general populations means, variances and heritabilities, are presented in table 1. Main comments are as it follows:

- $\mathrm{B}$ and $\mathrm{R}$ have practically the same 1000 seeds-weight.

- Progenies of $\mathrm{R}$ are more late flushing and more vigourous (dimensions, number of branches) than progenies of $B$.

- The between progenies variance is larger in B than in $R$. It is the contrary for the within progenies variance. Then, heritabilities for $\mathrm{B}$ are always larger than for $\mathrm{R}$. In most of cases, heritabilities for $\mathrm{R}$ are zero.

- Vigour and late flushing are positively correlated on phenotypic (in B and R, table 2) and genetic level (in B only).

The interpretation of this results leads to two types of hypothesis, one about the eventual influence of seed-trees age, the other one about genetical structure modifications. This last type of hypothesis explains more completely the differences between the mean progenies of $B$ and $R$.

- If the age of the seed-trees influences the seedlings vigour, in this case it is not through the seed-weight: it is necessary to imagine a modification of biochimical nature of the seed store, ARN, or cytoplasmical inheritance.

- The increase of vigour of $\mathrm{R}$ and the corresponding decrease of the additive genetical part of the total variance can be interpreted as an heterosis effect. This heterosis effect could 
result from the breakage of inbreeding circles in B caused by the long distance dispersion of seeds.

- It is possible to explain the increase of the lateness of flushing of $\mathrm{R}$ and the corresponding decrease of the between progenies variance by the action of a strong and early natu$\mathrm{ral}$ selection in favour of the late flushing individuals. The station of $\mathrm{R}$ is in fact specially exposed to spring frosts.

On a practical point of view, we can conclude:

- The settling of certain special micro-ecological aiea (spring frost hole for example) can be followed by a rough but localized evolution of the forest tree populations, caused by natural selection. It is necessary to take count of such facts for the sampling of a forest trees provenance.

- On account of the breakage of the inbreeding circles in the first generation of artificial stands and of the heterosis effect which can result occasionally in the second generation, it seems better to use these stands as seed sources rather than natural stands of the same phenotypic quality. On the contrary, it seems dangerous to select plus trees for rapid growth in artificial stands of second generation, because the superiority of these plus trees can mainly result from non additive genetical effects (overdominance).

- The flushing heritability in B $(0,40)$ shows that the selection of late flushing trees in natural forests, can lead to appreciable gain in flushing lateness. This gain in flushing lateness can be followed by an indirect increase of vigour (positive genetic correlation between late flushing and vigour).

\section{ZUSAMMENFASSUNG}

VERGLEICHVONZWEI AUFEINANDERFOLGENDER WEISSTANNENGENERATIONEN

( ABIES ALBA» MILL.). GENETISCHE STRUKTUR DER POPULATIONEN, HYPOTHESEN ÜBER DIE AKTION DER NATÜRLICHEN SELEKTION

Im Pflanzgarten wurden die mütterlichen Nachkommenschaften von 20 Bäumen eines 130 bis 140 jährigen autochtonen Tannenbestandes (B) sowie 20 Bäume einer aus B hervorgegangenen 40 jährigen Naturverjüngung (R) untersucht.

In der Tabelle 1, sind die Mittelwerte, Varianzen und Heritabilitäten der beiden Populationen wiedergeben und man kann daraus folgendes entnehmen:

- Das Tausendkorngewicht der Populationen B und $R$ ist nahezu gleich.

- Die Nachkommenschaften der Population $\mathrm{R}$ haben sowohl einen späteren vegetativen Austriebtermin als auch eine stärkere «Wuchskraft 》 (Pflanzendimensionen, Auzahl der Âste) als jene der Population B.

Die Variabilität zwischen den Familien (inter-famille) ist grösser bei der Population B. Bei der Variabilität innerhalb der Familien (intra famille) zeigt sich eine entgegengesetzte Tendenz. Daraus folgt, dass die Heritabilitätswerte der Population B ständig höher liegen als jene der Population R. Übrigens sind in der Mehrzahl der Fälle die Heritabilitätswerte der Population $\mathrm{R}$ gleich null.

In den Tabellen 2 und 3 sind die Korrelationen zwischen den veschiedenen beobachteten Merkmalen wiedergegeben. Dabei zeigt sich, dass insbesondere die Merkmale «Wuchskraft 》 (vigueur) und Spättreiben positiv korreliert sind und zwar sowohl in phänotypischer (B und R) als auch in genetischer Hinsicht (nur B).

Die Interpretierung der vorliegenden Ergebnisse führt zur Annahme von zwei Hypothesen. Die eine Hypothese betrifft den eventuellen Einfluss des Alters der Samenbäume, während die Andere auf Veränderungen der genetischen Struktur beruht. Letztere erlaubte eine befriedigendere Erklärung der festgestellen Unterschiede.

- Sollte das Alter der Samenbäume einen Einfluss auf die «Wuchskraft» der Sämlinge haben, so erfolgt dies im vorliegenden Fall nicht über das Tausendkorngewicht: man könnte eine biochemische Veränderung der Reservestoffe der Same-, oder des Zytoplasma-Erbgutes oder des ARN annehmen. 
— Die Erhöhung der «Wuchskraft» von $\mathrm{R}$ in Bezug auf $\mathrm{B}$ und die daraus folgende Verminderung des Anteils der additiven genetischen Varianz an der Gesamtvarianz kann auf einen Heterosis-Effekt zurückgeführt werden. Dieser Heterosis-Effekt könnte auf eine Unterbrechung der Inzuchtkreise der Population B zuruckgeführt werden, welche durch dié Saménverbreitung auf grössere Distanz hervorgerufen wird.

- Der spätere Autriebtermin der Population $\mathrm{R}$ und die entsprechende Abnahme der Variabilität zwischen den Familien (inter-famille), lässt sich durch eine brutale natürliche Auslese im Jugendstadium zum Vorteil der Spättreiber erklären.

Zusammenfassend lässt sich für die Praxis folgendes festhalten :

- Die Besiedlung bestimmter Mikrostdorte (z. B. Frostlöcher) kann mit einer brutalen jedoch lokalen Evolution der Populationen verbunden sein, welche durch eine natürliche Auslese hervorgerufen wind. Dies muss bei der Auswahl einer Provenienz berücksichtigt werden.

- Auf Grund der Unterbrechung der Inzuchtkreise in den nicht autochtonen Beständen erster Generation und dem eventuel daraus folgenden Heterosiseffekt, scheint es angebracht diese Besände den autochtonen Beständen gleicher phänotypischer Qualitât, zur Samengewinnung vorzuziehen. Dem gegenüber scheint es jedoch gefährlich in diésén Béständén Plusbäume bezüglich Zuchwachs auszuwàhlen, da deren Überlegenheit vorwiegend auf einen nicht additiven Heriditätseffekt (Superdominanz) beruhen kann.

- Die Heritabilität des Austreibens der Population B $(0,40)$ zeigt, dass die Auswahl spättreibender Bäume in einem autochtonen Bestand $\mathrm{zu}$ einem bemerkenswerten Gewinn führen kann. Dieser Gewinn der Austriebverzögerung kann mit einer indirekten Erhöhung der «Wuchskraft » verbunden sein (Positive genetische Korrelation zwischen «Spättreiben» und « Wuchskraft»).

\section{REFERENCES BIBLIOGRAPHIQUES}

Barber H. N., Jackson W. D., 1957. Natural selection in action in Eucalyptus. Nature, 179, 1267-69. Becker W. A., 1967. Manual of procedures in quantitative genetics. $2^{\mathrm{e}}$ édition. Washington State University Press. Washington. 7-10 et 69-79.

FALCONER D. S., 1964. Introduction to quantitative genetics. $3^{e}$ édition. Oliver and Boyd. Edinburgh and London. 150-183, 312-318.

Galoux A., 1969. Diversification génécologique régionale chez les espèces ligneuses feuillues. EEcol. Plant., 4, 1-14.

Hattemer H. H., 1963. Estimates of heritability published in forest tree breeding research. Proc. World Consultation Forest Genet. Tree Improvement., FAO/FORGEN 63-2a/3, 14 pp.

LACAZE J.-F., 1969. Etude de la variabilité infraspécifique de l'épicéa (Picea abies Karst.). - Provenances françaises et polonaises. Résultats au stade juvénile. Ann. Sci. forest., 26, (3), 345-396.

LANGNer W., 1953. Eine Mendelspaltung bei Aurea-Formen von Picea abies Karst. als Mittel zur Klärung der Befruchtungsverhãltnisse im Walde. Z. Forstgenetik, 3, 49-51.

ReEve E.C. R., 1955. The variance of the genetic correlation coefficient. Biometrics, 11, 357-374.

Arbez M., 1969. Etude comparée en pépinière de quelques provenances françaises de Sapin pectiné (Abies alba Mill.) - Premier aperçu de la variabilité infraspécifique et mise au point sur le «sapin de l'Aude». Ann. Sci. forest., 26, (4), 475-509. 